\title{
Bridging mathematics and physics: models of the evolution of dynamic aperture in hadron colliders and applications to LHC
}

\author{
F.F. Van der Veken ${ }^{* a, b}$, A. Bazzani ${ }^{c, d}$, M. Giovannozzi ${ }^{a}$, E.H. Maclean ${ }^{a, b}$, \\ C.E. Montanari ${ }^{c}$, and W. Van Goethem ${ }^{a, e}$ \\ ${ }^{a}$ CERN, Beams Department \\ 1211 Meyrin, Switzerland \\ ${ }^{b}$ University of Malta, Department of Communications \& Computer Engineering \\ MSD2080, Msida, Malta \\ ${ }^{c}$ Università di Bologna, Dipartimento di Fisica e Astronomia \\ 40126, Bologna, Italy \\ ${ }^{d}$ INFN sezione di Bologna \\ 40126, Bologna, Italy \\ ${ }^{e}$ Universiteit Antwerpen, Departement Fysica \\ 2020, Antwerp, Belgium
}

E-mail: frederik.van.der.veken@cern.ch

\begin{abstract}
When designing a high-energy, circular accelerator, like the upcoming High-Luminosity LHC or the future FCC, it is essential to have a reliable estimate of the expected beam losses and beam lifetime. A good prediction of the beam losses is essential to anticipate potential issues leading to quenches of the superconducting magnets or damage to the collimation system, while the beam lifetime is in direct relation to luminosity and, hence, to the overall performance of the accelerator. It is customary to make these estimations by means of the so-called dynamic aperture, which gives the extent of phase space in which the beam motion remains bounded for a given amount of time. The computational time for the evaluation of dynamic aperture has reached challenging levels, and as result the dynamic aperture can only be computed over a lapse of time that is too short with respect to the actual physical time scales. In this framework, intense efforts have been devoted to finding means of extrapolating the results of numerical simulations to more realistic time scales. The proposed approach is based on the Nekhoroshev theorem and Kolmogorov-Arnold-Moser theory of dynamical systems. This approach has provided the solid ground of well-established mathematical results to tackle a long-standing problem in accelerator physics. The technique has since been studied and tested in detail, both in numerical simulations and in experiments at the CERN Large Hadron collider with very encouraging results.
\end{abstract}

European Physical Society Conference on High Energy Physics - EPS-HEP2019 -

10-17 July, 2019

Ghent, Belgium

${ }^{*}$ Speaker. 


\section{Introduction}

One of the main concepts in the domain of single-particle beam dynamics is that of Dynamic Aperture (DA), representing the size of the connected phase-space volume exhibiting bounded motion for a given amount of time. DA is the key observable to guide the design of several past (see, e.g. [1-3]), present (see, e.g. [4]), and future (see e.g. [5-7]) circular hadron accelerators.

The DA is estimated using numerical simulations by tracking a set of initial conditions over a given number of turns around the ring. By flagging a particle as lost once its amplitude becomes too large, and by registering the time it took for the particle to get lost, an accurate approximation of the DA and its time evolution is recreated. Estimates of the magnetic field imperfections and misalignment errors of the machine are known within a certain uncertainty. To get a more robust estimate of the DA, several different machine realisations (so-called seeds), each with different errors sampled from the expected distributions, are used.

One of the main challenges when performing DA studies is their very CPU-intensive character, putting a strong limit on the time span that can be simulated. For the case of the CERN Large Hadron Collider (LHC), a typical upper limit is to track up to $10^{6}$ turns (representing only about $89 \mathrm{~s}$ of revolutions around the ring), whereas a typical fill time is of the order of several hours. A reliable model for the time evolution of the DA would allow to overcome the computational limitations in terms of CPU-time, opening the possibility to study observables that are more directly linked with the machine's performance, such as beam losses and lifetime [8].

Several attempts were made in earlier work $[9,10]$ to create models for the time evolution of DA. These were driven by fundamental results of dynamical system theory, such as the Kolmogorov-Arnold-Moser (KAM) [11 - 14] theory and the Nekhoroshev [15-18] theorem. Although very successful, this approach revealed two issues: the risk of obtaining nonphysical model parameters $[8,10]$ and the presence of internal dependencies among them [19-21].

An in-depth review has been carried out [22], the outcome of which is that it is indeed possible to overcome these two limitations. This is achieved by proposing a scaling law that is only based on Nekhoroshev theorem, and by using the parameters' dependencies obtained from a closer inspection of the form of the estimate of the stability time.

\section{Nekhoroshev theorem and different models of DA evolution}

The Nekhoroshev theorem provides an estimate for the number of turns $N(r)$ for which the orbit of an initial condition of amplitude $r$ remains bounded [16-18], namely

$$
\frac{N(r)}{N_{0}}=\sqrt{\frac{r}{r_{*}}} \exp \left\{\left(\frac{r_{*}}{r}\right)^{\frac{1}{\kappa}}\right\}
$$

where $r_{*}, N_{0}$, and $\kappa$ are positive quantities. As $r$ is the amplitude in which the phase space-orbits remain bounded, it is a literal representation of the DA. In earlier work $[9,10]$ a model was created by neglecting the square root, which is valid for large $r$, and by inverting Eq. (2.1)

$$
D A(N)=\frac{b}{\ln ^{\kappa} N}+D_{\infty}, \quad \text { Model } 1
$$


where $D_{\infty}$ is an asymptotic limit inspired by KAM theory, $N_{0}$ is set to 1 , and $b$ is just another notation for $r_{*}$. This model has been applied to data and thoroughly tested and generally has provided good results, but as mentioned above, also has a few issues. One can vary the way Eq. (2.1) is inverted, leading to different models as detailed in [22]. A first logical step to make, is to still neglect the square root, but to keep $N_{0}$ as a variable and remove $D_{\infty}$, hence constructing a model that is solely inspired on Nekhoroshev theorem, i.e.

$$
D A(N)=\frac{b}{\ln ^{\kappa} \frac{N}{N_{0}}} . \quad \text { Model } 2
$$

Next, it is possible to invert Eq. (2.1) exactly using the exotic Lambert-W function $\mathscr{W}:{ }^{1}$

$$
D A(N)=b\left[-\frac{\kappa}{2} \mathscr{W}_{-1}\left(-\frac{2}{\kappa}\left(\frac{N}{N_{0}}\right)^{-\frac{2}{\kappa}}\right)\right]^{-\kappa} \cdot \quad \text { Model } 4
$$

It might be advantageous to use the first order of the asymptotic expansion of $\mathscr{W}$ :

$$
D A(N)=b\left[\ln \frac{N}{N_{0}}+\frac{\kappa}{2}\left(\ln \frac{\kappa}{2}+\ln \left(\ln \frac{\kappa}{2}+\frac{2}{\kappa} \ln \frac{N}{N_{0}}\right)\right)\right]^{-\kappa} . \quad \text { Model } 3
$$

These models can be cast into different forms by using different definitions of the parameters to avoid internal dependencies [22]. The different definitions are summarised here for later reference:

$$
r_{*}=b \quad \rho=b\left(\frac{\kappa}{2}\right)^{-\kappa} \quad \rho_{*}=\rho \mathrm{e}^{\kappa} \quad \hat{\rho}_{*}=\frac{\rho_{*}}{6}
$$

Furthermore, there exists an estimate for $N_{0}$ from the original Nekhoroshev theorem:

$$
N_{0}=\frac{7}{8} \sqrt{\frac{b}{6}}
$$

This can be safely used for Model 4 and Model 3. For Model 2 the scaling in $b$ disappears and only a constant term remains; however it is not clear if this is correctly normalised. It is hence safer, in the case of Model 2, to set $N_{0}=1$ when not using it as a fit parameter.

The new models have been applied to a simple dynamical system like the 4D Hénon map, and to realistic realisations of the LHC ring at top energy, in the most stringent conditions, i.e. $N_{0}$ is not fitted, but set to 1 for Model 2 and to Eq. (2.7) for Models 3 and 4.

\section{Applying the different models to the Hénon map}

The modulated 4D Hénon map is given by

$$
\left(\begin{array}{c}
x \\
p_{x} \\
y \\
p_{y}
\end{array}\right)_{(n+1)}=\left(\begin{array}{cccc}
\cos \omega_{x}(n) & -\sin \omega_{x}(n) & 0 & 0 \\
\sin \omega_{x}(n) & \cos \omega_{x}(n) & 0 & 0 \\
0 & 0 & \cos \omega_{y}(n) & -\sin \omega_{y}(n) \\
0 & 0 & \sin \omega_{y}(n) & \cos \omega_{y}(n)
\end{array}\right)\left(\begin{array}{c}
x \\
p_{x}+x^{2}-y^{2} \\
y \\
p_{y}-2 x y
\end{array}\right)_{(n)}
$$

\footnotetext{
${ }^{1}$ For the numbering of the models we use the same ordering as in [22], hence Model 4 coming before Model 3.
} 
where $\left(x, p_{x}, y, p_{y}\right)$ are the phase-space co-ordinates after transformation to linear normalised coordinates and after rescaling by the strength of the sextupole, $\omega_{x}(n), \omega_{y}(n)$ read

$$
\omega_{x, y}(n)=\omega_{0 x, y}\left(1+\varepsilon \sum_{k=1}^{m} \varepsilon_{k} \cos \left(\Omega_{k} n\right)\right), \quad \omega_{0 x}=0.168, \quad \omega_{0 y}=0.201,
$$

and $\Omega_{k}$ and $\varepsilon_{k}$ are listed in [22].

Tracking has been performed up to $10^{7}$ turns, and the results of the first two models are shown in Fig. 1. Models 3 and 4 gave results that are indistinguishable by eye from Model 2 and are hence not shown. Model 1 reproduces the data more accurately than the new models. However, Model 1

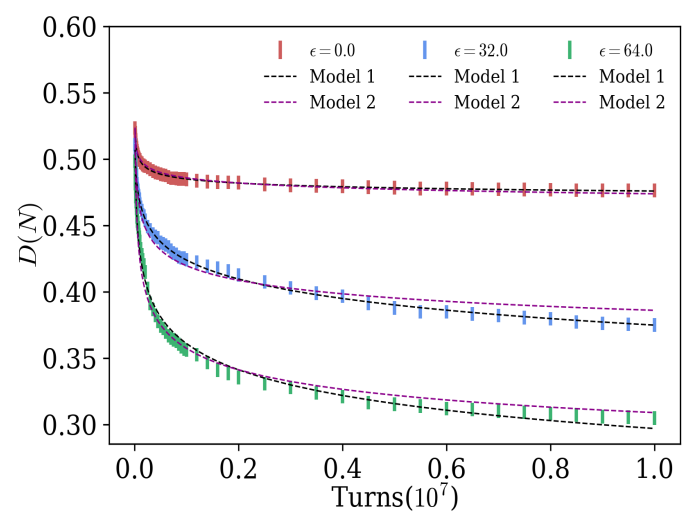

Figure 1: Markers: Evolution of DA as a function of turn number for different $\varepsilon$. The size of each marker represents the error associated with the DA. Lines: results of the DA Models 1 and 2.

has one extra fit parameter compared to the new models. It is instructive to inspect the dependence of the fit parameters on $\varepsilon$, as shown in Fig. 2. For Model 1, it is clearly visible that $\kappa$ and $b$ are
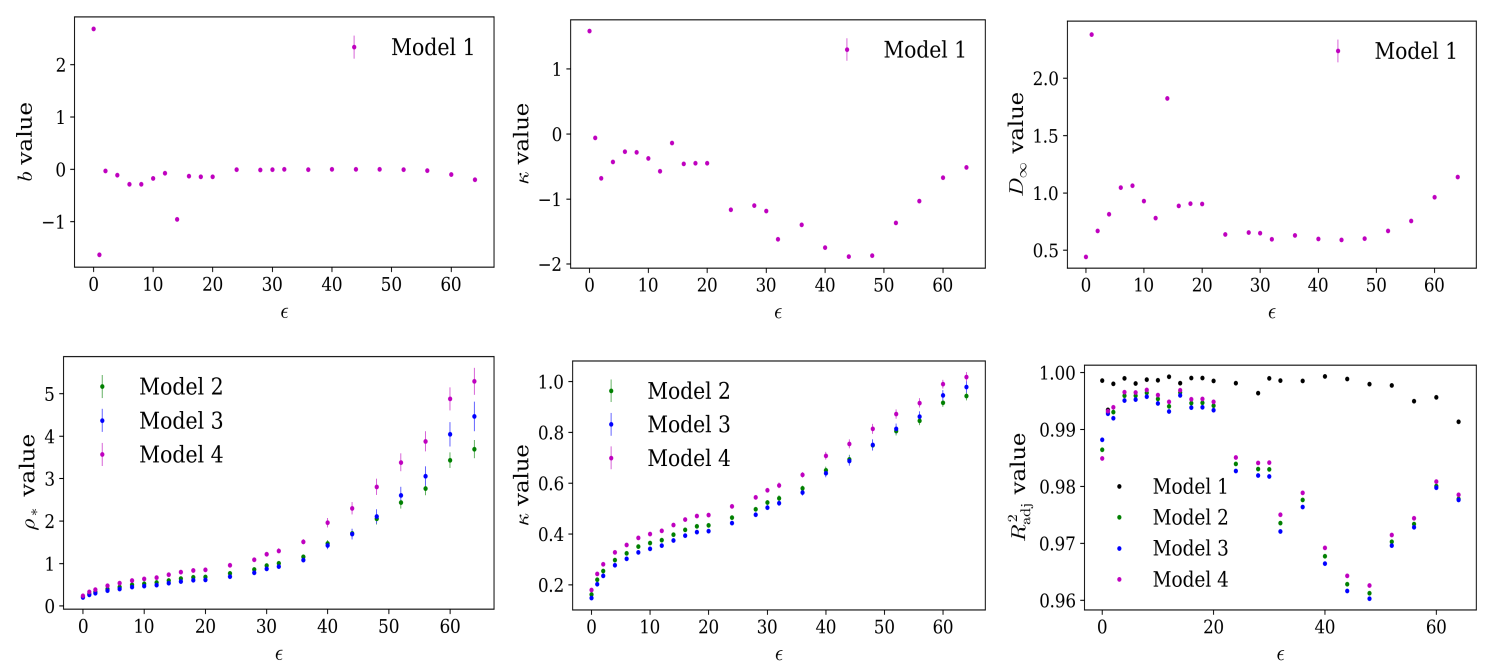

Figure 2: Dependence of the model parameters on $\varepsilon$ for Model 1 (upper) and the other models (lower). The figure of merit of the model quality, $R_{\mathrm{adj}}^{2}$, is also reported (lower right).

varying in sign, with rather larger changes of their absolute values. Such large variations are also 
visible in $D_{\infty}$. On the other hand, the three new models feature a rather smooth dependence on $\varepsilon$ and are very similar to each other.

\section{Applying the different models to the LHC}

DA simulations for the LHC are performed using the SixTrack code [23], by evolving initial conditions through the LHC magnetic lattice in $6 \mathrm{D}$. Several variations of the lattice configurations have been investigated, and the performance of the various DA models is largely independent on the specific configuration considered [22]. Therefore, the results from a single configuration are reported here.

Fig. 3 shows both the simulated DA and the value returned by the models. Moreover, the models have been used to extrapolate the DA up to $10^{8}$ turns. The bars reported in the four plots
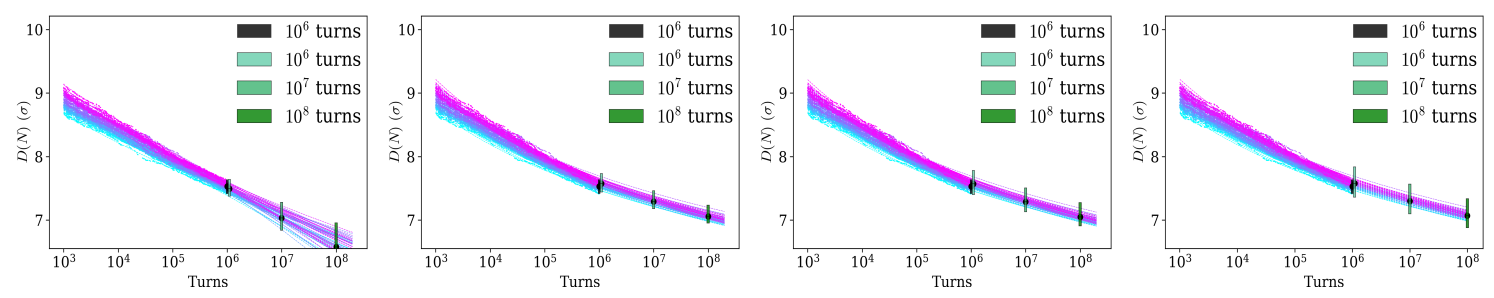

Figure 3: DA vs time for Models 1, 2, 3, and 4 (left to right). The various curves refer to the sixty realisations used in the numerical simulations, which are differentiated by their colour and stop at $10^{6}$ turns. Extrapolated values up to $10^{8}$ turns are also given, based on the models. The black bar indicates the DA from numerical simulations and its related uncertainty, whereas the green bars indicate the DA from the models.

are centred around the weighted average of the DA. The data from SixTrack is averaged over the different realisations, and the rms is used as the associated error. For the DA values obtained from the models, for each of the sixty realisations a model is fitted and the errors on the corresponding model parameters are used to evaluate the error associated with the DA estimate. Finally, all sixty DA values are averaged using the corresponding errors as weights, and the rms is used as associated error. These values are listed in Tab. 1. It is clear (both from Fig. 3 and Tab. 1) that the error bars for Model 1 are larger than those of the other models, thus confirming a better precision of the extrapolated DA values for Models 2, 3, and 4.

Table 1: DA values as obtained from simulations compared to DA as given by the four models.

\begin{tabular}{cccccc}
\hline & Turns & Model 1 & Model 2 & Model 3 & Model 4 \\
\hline \multirow{2}{*}{ Data } & $10^{6}$ & \multicolumn{4}{c}{$7.52 \pm 0.06_{-1.53 \%}^{+1.50 \%}$} \\
\hline \multirow{2}{*}{ Models } & $10^{6}$ & $7.50 \pm 0.05_{-1.60 \%}^{+1.94 \%}$ & $7.57 \pm 0.05_{-1.72 \%}^{+2.11 \%}$ & $7.57 \pm 0.05_{-2.24 \%}^{+2.79 \%}$ & $7.57 \pm 0.06_{-2.89 \%}^{+3.43 \%}$ \\
\cline { 2 - 6 } & $10^{7}$ & $7.03 \pm 0.09_{-2.78 \%}^{+3.51 \%}$ & $7.29 \pm 0.05_{-1.59 \%}^{+2.34 \%}$ & $7.29 \pm 0.05_{-2.12 \%}^{+3.02 \%}$ & $7.29 \pm 0.06_{-2.77 \%}^{+3.64 \%}$ \\
\cline { 2 - 6 } & $10^{8}$ & $6.6 \pm 0.2_{-6.37 \%}^{+5.77 \%}$ & $7.06 \pm 0.05_{-1.50 \%}^{+2.54 \%}$ & $7.05 \pm 0.05_{-2.02 \%}^{+3.21 \%}$ & $7.06 \pm 0.06_{-2.66 \%}^{+3.82 \%}$ \\
\hline \hline
\end{tabular}


Similar to the case for the Hénon map, Model 1 provides parameters that are often negative and vary significantly between the different realisations, unlike the new models, where all fit parameters are positive and their distribution features a narrow spread around their average value.

\section{Conclusions}

An active field in the domain of non-linear beam dynamics, aimed at providing accurate models, based on on fundamental results of the theory of non-linear dynamical systems, of the time variation of the extend of the DA has been discussed.

The very form of the original model, with the constant term $D_{\infty}$ that was inspired by KAM theory, turned out to be responsible for the appearance of negative nonphysical model parameters, and for having a larger variability of the model parameters. These issues are completely absent in the new proposed models. We would like to underline that the heart of the new models is the stability time estimate provided by the Nekhoroshev theorem, thus showing how mathematics is of paramount important to provide efficient solutions to physical problems.

This is an important step forward for a reliable description of DA, and hence for the possibility to extrapolate time-limited numerical computations to realistic time scales.

\section{References}

[1] N. M. Gelfand, Calculations of the dynamic aperture at the Tevatron, in Proc. of SSC Workshop on Accelerator Physics Issues for a Superconducting Super Collider, Ann Arbor (MI), USA, (1984) 124.

[2] F. Zimmermann and F. Willeke, Long term stability and dynamic aperture of the HERA proton ring, Tech. Rep. DESY-HERA-91-08, DESY, 1991.

[3] Y. Luo et al., Dynamic Aperture Evaluation at the Current Working Point for RHIC Polarized Proton Operation, in Proc. 22nd Particle Accelerator Conf. (PAC'07), Albuquerque (NM), USA, (2007) 4363.

[4] O. S. Brüning, P. Collier, P. Lebrun, S. Myers, R. Ostojic, J. Poole et al., LHC Design Report, CERN Yellow Rep. Monogr. CERN, Geneva, 2004, 10.5170/CERN-2004-003-V-1.

[5] Y. C. Jing, V. Litvinenko and D. Trbojevic, Optimization of Dynamic Aperture for Hadron Lattices in eRHIC, in Proc. 6th Int. Particle Accelerator Conf. (IPAC'15), Richmond (VA), USA, (2015) 757.

[6] G. Apollinari, I. Béjar Alonso, O. Brüning, P. Fessia, M. Lamont, L. Rossi et al., High-Luminosity Large Hadron Collider (HL-LHC), vol. 4 of CERN Yellow Rep. Monogr. CERN, Geneva, 2017, 10.23731/CYRM-2017-004.

[7] E. Cruz Alaniz et al., Methods to increase the dynamic aperture of the FCC-hh lattice, in Proc. 9th Int. Particle Accelerator Conf. (IPAC'18), Vancouver, Canada, (2018) 3593.

[8] M. Giovannozzi, A proposed scaling law for intensity evolution in hadron storage rings based on dynamic aperture variation with time, Phys. Rev. ST Accel. Beams 15 (2012) 024001.

[9] M. Giovannozzi, W. Scandale and E. Todesco, Prediction of long-term stability in large hadron colliders, Part. Accel. 56 (1997) 195.

[10] M. Giovannozzi, W. Scandale and E. Todesco, Dynamic aperture extrapolation in presence of tune modulation, Phys. Rev. E57 (1998) 3432. 
[11] A. N. Kolmogorov, On the conservation of conditionally periodic motions under small perturbation of the hamiltonian, Dokl. Akad. Nauk SSR 98 (1954) 527.

[12] J. Moser, On invariant curves of area-preserving mappings of an annulus, Nachr. Akad. Wiss. Göttingen Math.-Phys. Kl. II (1962) 1.

[13] V. Arnold, Proof of a theorem of a.n. kolmogorov on the preservation of conditionally periodic motions under a small perturbation of the hamiltonian, Russ. Math. Surv. 18 (1963) 9.

[14] C. L. Siegel and J. Moser, Lectures in celestial mechanics. Berlin Springer Verlag, Berlin, Germany, 1971.

[15] N. Nekhoroshev, Behavior of hamiltonian systems close to integrable, in Functional Analysis and Its Applications, vol. 5, p. 338, Kluwer Academic Publishers-Plenum Publishers, (1971), DOI.

[16] N. Nekhoroshev, An exponential estimate of the time of stability of nearly-integrable Hamiltonian systems, Russ. Math. Surv. 32 (1977) 1.

[17] A. Bazzani, S. Marmi and G. Turchetti, Nekhoroshev estimate for isochronous non resonant symplectic maps, Cel. Mech. 47 (1990) 333.

[18] G. Turchetti, Nekhoroshev stability estimates for symplectic maps and physical applications, in Proc. of the Winter School, vol. 47 of Springer Proceedings in Physics, Les Houches, France ('89), (1990) 223.

[19] M. Giovannozzi and F. F. Van der Veken, Description of the luminosity evolution for the CERN LHC including dynamic aperture effects. Part I: the model, Nucl. Instrum. Methods Phys. Res. $\mathbf{A 9 0 5}$ (2018) $171[1806.03058]$.

[20] M. Giovannozzi and F. F. Van der Veken, Description of the luminosity evolution for the CERN LHC including dynamic aperture effects. Part II: application to Run 1 data, Nucl. Instrum. Methods Phys. Res. A908 (2018) 1 [1806.03059].

[21] F. F. Van der Veken and M. Giovannozzi, Scaling Laws for the Time Dependence of Luminosity in Hadron Circular Accelerators based on Simple Models of Dynamic Aperture Evolution, in Proc. 61st ICFA Advanced Beam Dynamics Workshop on High-Intensity and High-Brightness Hadron Beams (HB'18), Daejeon, Korea, (2018) 260.

[22] A. Bazzani, M. Giovannozzi, E. H. Maclean, C. E. Montanari, F. F. Van der Veken and W. Van Goethem, Advances on the modeling of the time evolution of dynamic aperture of hadron circular accelerators, Phys. Rev. Accel. Beams 22 (2019) 104003 [1909. 09516].

[23] R. De Maria et al., "SixTrack - 6D Tracking Code.” Available at http://sixtrack.web.cern.ch/SixTrack/. 